\title{
DIVISION III / WORKING GROUP PLANETARY SYSTEM NOMENCLATURE
}

\author{
LA NOMENCLATURE DU SYSTÈME PLANÉTAIRE
}

CHAIR

MEMBERS

\author{
Rita M. Schulz \\ Kaare Aksnes, Jennifer S. Blue, \\ Jürgen Blunck ( $†$ July 2008), \\ Edward L. G. Bowell, George A. Burba, \\ Guy J. Consolmagno (since November 2008), \\ Régis Courtin, Rosaly M. Lopes-Gautier, \\ Mikhail Ya. Marov, Brian G. Marsden, \\ Mark S. Robinson, Vladislav V. Shevchenko, \\ Bradford A. Smith
}

\section{TRIENNIAL REPORT 2006 - 2009}

\section{Introduction}

The Working Group on Planetary System Nomenclature (WG-PSN) develops, maintains and publishes guidelines for naming natural satellites of planets and surface features on all solar system bodies except Earth. When required the WG approves lists of new nomenclature, with accompanying explanatory notes, based on the established guidelines. Approved names are immediately added into the Gazetteer of Planetary Nomenclature. Objections based on significant, substantive problems may be submitted within a 3-months period, and will be ruled on by Division III.

The WG-PSN is supported by six Tasks Groups dedicated to naming issues for the Moon, Mercury, Venus, Mars, Outer Solar System, and Small Bodies. To simplify the name-request process for the scientific community a Name Request Form has been made available electronically on the Gazetteer web page. This also ensures that a data archive of name requests can be maintained by the Working Group. A page with frequently asked questions (FAQ) was also added to the Gazetteer. Details on the nomenclature and the naming process can be obtained from the Gazetteer of Planetary Nomenclature $<$ planetarynames.wr.usgs.gov>.

\section{Activities between the IAU XXVI General Assembly in 2006 and 1 June 2008}

Since the IAU General Assembly in Prague in August 2006, all discussion and approval procedures were handled via email. As of 1 June 2008, the WG-PSN has approved 259 new names for surface features on solar system bodies: 46 on Dione, 35 on Enceladus, 9 on Europa, 12 on Mercury, 2 on Io, 79 on Mars, 8 on Phobos, 33 on Tethys, 22 on Titan, and 13 on Venus. In addition 22 names for satellites of the Giant Planets were approved, 1 of Jupiter, 17 of Saturn, and 4 of Neptune, and the spelling of one Saturnian satellite was corrected. Concerning ring systems, the WG-PSN provided the following definition 
for divisions and gaps: 'Divisions are the separations between named rings, and gaps are the spaces within named rings. In general, divisions are large, gaps are small.' In accordance with this definition the 'Roche Division' of the Saturnian ring system was defined as the division between the outer edge of the A ring and the inner edge of the $\mathrm{F}$ ring, and the term 'Encke Division' was changed to 'Encke Gap'. In addition, new figures for Saturn's rings were approved and added to the Gazetteer.

A rule for the procedure of dropping names became necessary, which was defined as follows.

"Official approval by a Task Group and by the Working Group is required before a name can be dropped. Dropped names are retained in the database for reference; the code in the 'approval status' field is changed to ' 6 ' indicating the name has been dropped, the name is shown in brackets in the 'name' field, and a brief notation is made in the 'origin' field explaining why the name was dropped and when it was dropped. In general, names that have been dropped should not be re-used for other features. Dropped names could be reused in very exceptional cases; for instance, if no new names of a particular theme are available and there is strong justification, a dropped name could be considered for reuse."

Six names were dropped, 4 on Mars, 1 on Venus, and 1 on Dione, for which the descriptor terms of three names were also revised.

The existing definition of the descriptor term 'mare/maria' (as used on the Moon) was amended for use on Titan and now reads: 'Sea; large circular plain; on Titan, large expanses of dark materials thought to be liquid hydrocarbons'. The descriptor term Insula/insulae was added with the definition: 'Island (islands), an isolated land area (or group of such areas) surrounded by, or nearly surrounded by, a liquid area (sea or lake)'. A few particular themes for Mars, Phobos, Mercury and Io were expanded and four new themes were added for Titan.

\section{Closing remarks}

The future activities will continue to be carried out mainly via email. However, a WGPSN meeting will be held in Ithaca, NY, USA on 10-11 October 2008, to discuss basic working group business, such as performance, need for rules and guidelines, and issues of concern. All members of the WG and the Task Groups have been invited to attend and to submit agenda items.

Rita M. Schulz

chair of the Working Group 